\title{
SOME REFINEMENTS AND GENERALIZATIONS OF CARLEMAN'S INEQUALITY
}

\section{DAH-YAN HWANG}

Received 24 November 2003

We give some refinements and generalizations of Carleman's inequality with weaker condition for weight coefficient.

2000 Mathematics Subject Classification: 26D15.

1. Introduction. The following Carleman's inequality (see [6, Theorem 334]) is well known, unless $\left(a_{n}\right)$ is null:

$$
\sum_{n=1}^{\infty}\left(a_{1} a_{2} \cdots a_{n}\right)^{1 / n}<e \sum_{n=1}^{\infty} a_{n} .
$$

The constant is the best possible.

There is a vast literature which deals with alternative proofs, various generalizations and extensions, and numerous variants and applications in analysis of inequality (1.1); see $[1,2,3,5,7,9,8,10,13,14,15,16,17,18,19]$ and the references given therein. According to Hardy (see [6, Theorem 349]), Carleman's inequality was generalized as follows. If $a_{n} \geq 0, \lambda_{n} \geq 0, \Lambda_{n}=\sum_{m=1}^{n} \lambda_{m}(n \in \mathbb{N})$, and $0<\sum_{n=1}^{\infty} \lambda_{n} a_{n}<\infty$, then

$$
\sum_{n=1}^{\infty} \lambda_{n}\left(a_{1}^{\lambda_{1}} a_{2}^{\lambda_{2}} \cdots a_{n}^{\lambda_{n}}\right)^{1 / \Lambda_{n}}<e \sum_{n=1}^{\infty} \lambda_{n} a_{n} .
$$

In [19], Yuan obtained the refined Carleman's inequality as follows. If $a_{n} \geq 0, n=$ $1,2, \ldots$, and $0<\sum_{n=1}^{\infty} a_{n}<\infty$, then

$$
\sum_{n=1}^{\infty}\left(a_{1} a_{2} \cdots a_{n}\right)^{1 / n}<e \sum_{n=1}^{\infty} \frac{(1-\beta / n)}{(1+1 / n)^{\alpha}} a_{n},
$$

where $\alpha, \beta$ satisfy $0 \leq \alpha \leq 1 / \ln 2-1,0 \leq \beta \leq 1-2 / e$, and $e \beta+2^{1+\alpha}=e$.

Recently, Kim [10] established the following new extension of the refined Hardy's inequality in the spirit of the property of the power mean of $n$ distinct positive numbers. 
THEOREM 1.1. If $0<\lambda_{n+1} \leq \lambda_{n}, a_{n} \geq 0, \Lambda_{n}=\sum_{m=1}^{n} \lambda_{m}, \Lambda_{n} \geq 1,0<p \leq 1$, and $0<\sum_{n=1}^{\infty} \lambda_{n} a_{n}<\infty$, then

$$
\begin{aligned}
& \sum_{n=1}^{\infty} \lambda_{n+1}\left(a_{1}^{\lambda_{1}} a_{2}^{\lambda_{2}} \cdots \lambda_{n}^{\lambda_{n}}\right)^{1 / \Lambda_{n}} \\
& \quad<\frac{e^{p}}{p} \sum_{n=1}^{\infty}\left(\frac{\Lambda_{n}^{\alpha}\left(\Lambda_{n}-\lambda_{n} \beta\right)}{\Lambda_{n}\left(\Lambda_{n}+\lambda_{n}\right)^{\alpha}}\right)^{p} \lambda_{n} a_{n}^{p} \Lambda_{n}^{p-1}\left(\sum_{k=1}^{n} \lambda_{k} c_{k}^{p} a_{k}^{p}\right)^{(1-p) / p},
\end{aligned}
$$

where $c_{k}^{\lambda k}=\left(\Lambda_{k+1}\right)^{\Lambda_{k}} /\left(\Lambda_{k}\right)^{\Lambda_{k-1}}$, and $\alpha, \beta$ satisfy $0 \leq \alpha \leq 1 / \ln 2-1,0 \leq \beta \leq 1-2 / e$, and $e \beta+2^{1+\alpha}=e$.

When $p=1$ in inequality (1.4), there exists the following class of new refined Hardy's inequality:

$$
\sum_{n=1}^{\infty} \lambda_{n+1}\left(a_{1}^{\lambda_{1}} a_{2}^{\lambda_{2}} \cdots a_{n}^{\lambda_{n}}\right)^{1 / \Lambda_{n}}<e \sum_{n=1}^{\infty}\left(\frac{\Lambda_{n}^{\alpha}\left(\Lambda_{n}-\lambda_{n} \beta\right)}{\Lambda_{n}\left(\Lambda_{n}+\lambda_{n}\right)^{\alpha}}\right) \lambda_{n} a_{n} .
$$

In this paper, we will establish some new refinements and generalizations of Carleman's inequality. Also, our results correspond to Theorem 1.1 with a weaker condition for weight coefficient.

2. Results. The following results are new generalizations of Carleman's inequality.

THEOREM 2.1. Let $\lambda_{n}>0, v_{n}>0, a_{n} \geq 0, \Lambda_{n}=\sum_{m=1}^{n} \lambda_{m} v_{m}(n \in \mathbb{N}), 0<p \leq 1$, and $0<\sum_{n=1}^{\infty} \lambda_{n} v_{n} a_{n}<\infty$. If

$$
\frac{\Lambda_{n+1} \Lambda_{n}}{\Lambda_{n}+\lambda_{n} \nu_{n}} \leq \Lambda_{n-1}^{(1-1 / p)\left(\Lambda_{n-1} / \Lambda_{n}\right)} \Lambda_{n}^{1 / p} v_{n}^{-\left(\Lambda_{n-1} / \Lambda_{n}\right)} v_{n+1}
$$

then

$$
\begin{aligned}
& \sum_{n=1}^{\infty} \lambda_{n+1}\left(a_{1}^{\lambda_{1} v_{1}} a_{2}^{\lambda_{2} v_{2}} \cdots a_{n}^{\lambda_{n} v_{n}}\right)^{1 / \Lambda_{n}} \\
& \quad \leq \frac{e^{p}}{p} \sum_{n=1}^{\infty}\left(\frac{\Lambda_{n}^{\alpha}\left(\Lambda_{n}-\lambda_{n} v_{n} \beta\right)}{\Lambda_{n}\left(\Lambda_{n}+\lambda_{n} v_{n}\right)^{\alpha}}\right)^{p} \lambda_{n} v_{n} a_{n}^{p} \Lambda_{n}^{p-1}\left(\sum_{k=1}^{n} \lambda_{k} v_{k}\left(c_{k} a_{k}\right)^{p}\right)^{(1-p) / p},
\end{aligned}
$$

where

$$
c_{k}=\left[\frac{\left(\Lambda_{k}^{(1-1 / p)} \Lambda_{k+1}\right)^{\Lambda_{k}} v_{k}^{\Lambda_{k-1}}}{\left(\Lambda_{k-1}^{(1-1 / p)} \Lambda_{k}\right)^{\Lambda_{k-1}} v_{k+1}^{\Lambda_{k}}}\right]^{1 / \lambda_{k} v_{k}}
$$

and $\alpha, \beta$ satisfy $0 \leq \alpha \leq 1 / \ln 2-1,0 \leq \beta \leq 1-2 / e$, and $e \beta+2^{1+\alpha}=e$. 
Proof. By the power mean inequality [11, page 15], we have

$$
\alpha_{1}^{q_{1}} \alpha_{2}^{q_{2}} \cdots \alpha_{n}^{q_{n}} \leq\left(\sum_{m=1}^{n} q_{m} \alpha_{m}^{p}\right)^{1 / p}
$$

for $\alpha_{m} \geq 0, p>0$, and $q_{m}>0(m \in \mathbb{N})$ with $\sum_{m=1}^{n} q_{m}=1$. Setting $c_{m}>0, \alpha_{m}=c_{m} a_{m}$, and $q_{m}=\lambda_{m} v_{m} / \Lambda_{n}$, we obtain

$$
\left(c_{1} a_{1}\right)^{\lambda_{1} v_{1} / \Lambda_{n}}\left(c_{2} a_{2}\right)^{\lambda_{2} v_{2} / \Lambda_{n}} \cdots\left(c_{n} a_{n}\right)^{\lambda_{n} v_{n} / \Lambda_{n}} \leq\left(\sum_{m=1}^{n} \frac{\lambda_{m} \nu_{m}}{\Lambda_{n}}\left(c_{m} a_{m}\right)^{p}\right)^{1 / p} .
$$

Using the above inequality, we have

$$
\begin{aligned}
& \sum_{n=1}^{\infty} \lambda_{n+1}\left(a_{1}^{\lambda_{1} v_{1}} a_{2}^{\lambda_{2} v_{2}} \cdots a_{n}^{\lambda_{n} v_{n}}\right)^{1 / \Lambda_{n}} \\
&\left.\quad=\sum_{n=1}^{\infty} \lambda_{n+1} \frac{\left(c_{1} a_{1}\right)^{\lambda_{1} v_{1} / \Lambda_{n}}\left(c_{2} a_{2}\right)^{\lambda_{2} v_{2} / \Lambda_{n}} \cdots\left(c_{n} a_{n}\right)^{\lambda_{n} v_{n} / \Lambda_{n}}}{\left(c_{1}^{\lambda_{1} v_{1}} c_{2}^{\lambda_{2} v_{2}} \cdots c_{n}^{\lambda_{n} v_{n}}\right)^{1 / \Lambda_{n}}}\right)\left(\frac{1}{\Lambda_{n}} \sum_{m=1}^{n} \lambda_{m} v_{m}\left(c_{m} a_{m}\right)^{p}\right)^{1 / p} . \\
& \quad \leq \sum_{n=1}^{\infty}\left(\frac{\lambda_{n+1}}{\left(c_{1}^{\lambda_{1} v_{1}} c_{2}^{\lambda_{2} v_{2}} \cdots c_{n}^{\lambda_{n} v_{n}}\right)^{1 / \Lambda_{n}}} .\right.
\end{aligned}
$$

By using the inequality (see [4, 12])

$$
\left(\sum_{m=1}^{n} z_{m}\right)^{t} \leq t \sum_{m=1}^{n} z_{m}\left(\sum_{k=1}^{m} z_{k}\right)^{t-1}
$$

where $t \geq 1$ is constant and $z_{m} \geq 0(m \in \mathbb{N})$, it is easy to observe that

$$
\left(\frac{1}{\Lambda_{n}} \sum_{m=1}^{n} \lambda_{m} \nu_{m}\left(c_{m} a_{m}\right)^{p}\right)^{1 / p} \leq \frac{1}{p \Lambda_{n}^{1 / p}} \sum_{m=1}^{n} \lambda_{m} v_{m}\left(c_{m} a_{m}\right)^{p}\left(\sum_{k=1}^{m} \lambda_{k} v_{k}\left(c_{k} a_{k}\right)^{p}\right)^{(1-p) / p}
$$

for $\Lambda_{n} \geq 1$ and $0<p \leq 1$. Then, by (2.6) and (2.8), we obtain

$$
\begin{aligned}
& \sum_{n=1}^{\infty} \lambda_{n+1}\left(a_{1}^{\lambda_{1} v_{1}} a_{2}^{\lambda_{2} v_{2}} \cdots a_{n}^{\lambda_{n} v_{n}}\right)^{1 / \Lambda_{n}} \\
& \leq \frac{1}{p} \sum_{n=1}^{\infty} \frac{\lambda_{n+1}}{\Lambda_{n}^{1 / p}\left(c_{1}^{\lambda_{1} v_{1}} c_{2}^{\lambda_{2} v_{2}} \cdots c_{n}^{\lambda_{n} v_{n}}\right)^{1 / \Lambda_{n}}} \sum_{m=1}^{n} \lambda_{m} v_{m}\left(c_{m} a_{m}\right)^{p}\left(\sum_{k=1}^{m} \lambda_{k} v_{k}\left(c_{k} a_{k}\right)^{p}\right)^{(1-p) / p} \\
& =\frac{1}{p} \sum_{m=1}^{n} \lambda_{m} v_{m}\left(c_{m} a_{m}\right)^{p} \sum_{n=m}^{\infty}\left(\frac{\lambda_{n+1}}{\Lambda_{n}^{1 / p}\left(c_{1}^{\lambda_{1} v_{1}} c_{2}^{\lambda_{2} v_{2}} \cdots c_{n}^{\lambda_{n} v_{n}}\right)^{1 / \Lambda_{n}}}\right)\left(\sum_{k=1}^{m} \lambda_{k} v_{k}\left(c_{k} a_{k}\right)^{p}\right)^{(1 / p) / p} .
\end{aligned}
$$


Choosing $c_{1}^{\lambda_{1} v_{1}} c_{2}^{\lambda_{2} v_{2}} \cdots c_{n}^{\lambda_{n} v_{n}}=\left(\Lambda_{n}^{(1-1 / p)} \Lambda_{n+1} v_{n+1}^{-1}\right)^{\Lambda_{n}}(n \in \mathbb{N})$ and setting $\Lambda_{0}=1$, from (2.1), it follows that

$$
\begin{aligned}
c_{n} & =\left(\frac{\left(\Lambda_{n}^{(1-1 / p)} \Lambda_{n+1} v_{n+1}^{-1}\right)^{\Lambda_{n}}}{\left(\Lambda_{n-1}^{(1-1 / p)} \Lambda_{n} v_{n}^{-1}\right)^{\Lambda_{n-1}}}\right)^{1 / \lambda_{n} v_{n}} \\
& =\left(\frac{\Lambda_{n+1}}{\Lambda_{n-1}^{(1-1 / p)\left(\Lambda_{n-1} / \Lambda_{n}\right)} \Lambda_{n}^{1 / p} v_{n}^{-\left(\Lambda_{n-1} / \Lambda_{n}\right)} \cdot v_{n+1}}\right)^{\Lambda_{n} / \lambda_{n} v_{n}} \Lambda_{n} \\
& \leq\left(1+\frac{\lambda_{n} v_{n}}{\Lambda_{n}}\right)^{\Lambda_{n} / \lambda_{n} v_{n}} \Lambda_{n} .
\end{aligned}
$$

This implies that

$$
\begin{aligned}
& \sum_{n=1}^{\infty} \lambda_{n+1}\left(a_{1}^{\lambda_{1} v_{1}} a_{2}^{\lambda_{2} v_{2}} \cdots a_{n}^{\lambda_{n} v_{n}}\right)^{1 / \Lambda_{n}} \\
& \leq \frac{1}{p} \sum_{m=1}^{n} \lambda_{m} v_{m}\left(c_{m} a_{m}\right)^{p} \sum_{n=m}^{\infty} \frac{\lambda_{n+1} v_{n+1}}{\Lambda_{n} \Lambda_{n+1}}\left(\sum_{k=1}^{m} \lambda_{k} v_{k}\left(c_{k} a_{k}\right)^{p}\right)^{(1-p) / p} \\
& =\frac{1}{p} \sum_{m=1}^{n} \lambda_{m} v_{m}\left(c_{m} a_{m}\right)^{p} \sum_{n=m}^{\infty}\left(\frac{1}{\Lambda_{n}}-\frac{1}{\Lambda_{n+1}}\right)\left(\sum_{k=1}^{m} \lambda_{k} v_{k}\left(c_{k} a_{k}\right)^{p}\right)^{(1-p) / p} \\
& =\frac{1}{p} \sum_{m=1}^{n} \lambda_{m} v_{m}\left(c_{m} a_{m}\right)^{p} \frac{1}{\Lambda_{n}}\left(\sum_{k=1}^{m} \lambda_{k} v_{k}\left(c_{k} a_{k}\right)^{p}\right)^{(1-p) / p} \\
& \leq \frac{1}{p} \sum_{m=1}^{n}\left(1+\frac{\lambda_{m} v_{m}}{\Lambda_{m}}\right)^{p \Lambda_{m} / \lambda_{m} v_{m}} \lambda_{m} v_{m} a_{m}^{p} \Lambda_{m}^{p-1}\left(\sum_{k=1}^{m} \lambda_{k} v_{k}\left(c_{k} a_{k}\right)^{p}\right)^{(1-p) / p}
\end{aligned}
$$

Hence, using the inequality [19, Lemma 3.1]

$$
\left(1+\frac{1}{x}\right)^{x} \leq e \frac{(1-\beta / x)}{(1+1 / x)^{\alpha}}
$$

for $x>1$, and $\alpha, \beta$ satisfying $0 \leq \alpha \leq 1 / \ln 2-1,0 \leq \beta \leq 1-2 / e$, and $e \beta+2^{1+\alpha}=e$, we have (2.2). Thus Theorem 2.1 is proved.

Taking $v_{n}=1(n \in \mathbb{N})$ in Theorem 2.1, we have the following corollary.

COROLlARY 2.2. Let $\lambda_{n}>0, a_{n} \geq 0, \Lambda_{n}=\sum_{m=1}^{n} \lambda_{m}(n \in \mathbb{N}), 0<p \leq 1$, and $0<$ $\sum_{n=1}^{\infty} \lambda_{n} a_{n}<\infty$. If

$$
\frac{\Lambda_{n+1} \Lambda_{n}}{\Lambda_{n}+\lambda_{n}} \leq \Lambda_{n-1}^{(1-1 / p)\left(\Lambda_{n-1} / \Lambda_{n}\right)} \Lambda_{n}^{1 / p}
$$


then

$$
\sum_{n=1}^{\infty} \lambda_{n+1}\left(a_{1}^{\lambda_{1}} a_{2}^{\lambda_{2}} \cdots a_{n}^{\lambda_{n}}\right)^{1 / \Lambda_{n}} \leq \frac{e^{p}}{p} \sum_{n=1}^{\infty}\left(\frac{\Lambda_{n}^{\alpha}\left(\Lambda_{n}-\lambda_{n} \beta\right)}{\Lambda_{n}\left(\Lambda_{n}+\lambda_{n}\right)^{\alpha}}\right)^{p} \lambda_{n} a_{n}^{p} .
$$

REMARK 2.3. Corollary 2.2 is a result corresponding to Theorem 1.1 with a weaker condition for $\lambda_{n}(n \in \mathbb{N})$. Further, setting $p=1$ in Corollary 2.2, we obtain an inequality corresponding to inequality (1.5) with a weaker condition for $\Lambda_{n}(n \in \mathbb{N})$.

Setting $p=1$ in Theorem 2.1, we obtain the following corollary.

COROLlARY 2.4. Let $\lambda_{n}>0, v_{n}>0, a_{n} \geq 0, \Lambda_{n}=\sum_{m=1}^{n} \lambda_{m} v_{m}(n \in \mathbb{N})$, and $0<$ $\sum_{n=1}^{\infty} \lambda_{n} v_{n} a_{n}<\infty$. If

$$
\frac{\Lambda_{n+1}}{\Lambda_{n}+\lambda_{n} \nu_{n}} \leq v_{n}^{-\left(\Lambda_{n-1} / \Lambda_{n}\right)} v_{n+1}
$$

then

$$
\sum_{n=1}^{\infty} \lambda_{n+1}\left(a_{1}^{\lambda_{1} v_{1}} a_{2}^{\lambda_{2} v_{2}} \cdots a_{n}^{\lambda_{n} v_{n}}\right)^{1 / \Lambda_{n}} \leq e \sum_{n=1}^{\infty}\left(\frac{\Lambda_{n}^{\alpha}\left(\Lambda_{n}-\lambda_{n} v_{n} \beta\right)}{\Lambda_{n}\left(\Lambda_{n}+\lambda_{n} \nu_{n}\right)^{\alpha}}\right) \lambda_{n} v_{n} a_{n}
$$

REMARK 2.5. Inequality (2.16) is a new refinement and generalization of inequality (1.3).

COROLLARY 2.6. Under the assumptions of Theorem 2.1, if $\alpha=0, \beta=1-2 / e$, then

$$
\begin{aligned}
& \sum_{n=1}^{\infty} \lambda_{n+1}\left(a_{1}^{\lambda_{1} v_{1}} a_{2}^{\lambda_{2} v_{2}} \cdots a_{n}^{\lambda_{n} v_{n}}\right)^{1 / \Lambda_{n}} \\
& \quad \leq \frac{e^{p}}{p} \sum_{n=1}^{\infty}\left(1-\frac{\lambda_{n} v_{n}(1-2 / e)}{\Lambda_{n}}\right)^{p} \lambda_{n} v_{n} a_{n}^{p} \Lambda_{n}^{p-1}\left(\sum_{k=1}^{n} \lambda_{k} v_{k}\left(c_{k} a_{k}\right)^{p}\right)^{(1-p) / p},
\end{aligned}
$$

where (2.3) holds.

REMARK 2.7. Corollary 2.6 is a new refinement and generalization of [10, Corollary 2.3]. Further, setting $v_{n}=1(n \in \mathbb{N})$ in Corollary 2.6, we obtain a result corresponding to [10, Corollary 2.3] with a weaker condition for $\lambda_{n}(n \in \mathbb{N})$.

COROLLARY 2.8. Under the assumptions of Theorem 2.1 , if $\alpha=1 / \ln 2-1, \beta=0$, then

$$
\begin{aligned}
& \sum_{n=1}^{\infty} \lambda_{n+1}\left(a_{1}^{\lambda_{1} v_{1}} a_{2}^{\lambda_{2} v_{2}} \cdots a_{n}^{\lambda_{n} v_{n}}\right)^{1 / \Lambda_{n}} \\
& \quad \leq \frac{e^{p}}{p} \sum_{n=1}^{\infty}\left(\left(1+\frac{\lambda_{n} v_{n}}{\Lambda_{n}}\right)^{1-1 / \ln 2}\right)^{p} \lambda_{n} v_{n} a_{n}^{p} \Lambda_{n}^{p-1}\left(\sum_{k=1}^{n} \lambda_{k} v_{k}\left(c_{k} a_{k}\right)^{p}\right)^{(1-p) / p},
\end{aligned}
$$

where (2.3) holds. 
REMARK 2.9. Corollary 2.8 is a new refinement and generalization of [10, Corollary 2.4]. Further, setting $v_{n}=1(n \in \mathbb{N})$ in Corollary 2.8, we obtain a result corresponding to [10, Corollary 2.4] with a weaker condition for $\lambda_{n}(n \in \mathbb{N})$.

A new general refined Hardy's inequality is introduced to the following theorem.

THEOREM 2.10. Let $\lambda_{n}>0, v_{n}>0, a_{n} \geq 0, \Lambda_{n}=\sum_{m=1}^{n} \lambda_{m} v_{m}(n \in \mathbb{N})$, and $0<$ $\sum_{n=1}^{\infty} \lambda_{n} v_{n} a_{n}<\infty$ for $0<p \leq t<\infty$. If

$$
\frac{\Lambda_{n+1} \Lambda_{n}}{\Lambda_{n}+\lambda_{n} v_{n}} \leq \Lambda_{n-1}^{((p-t) / p)\left(\Lambda_{n-1} / \Lambda_{n}\right)} \Lambda_{n}^{t / p} v_{n}^{-\left(\Lambda_{n-1} / \Lambda_{n}\right)} v_{n+1}
$$

then

$$
\begin{aligned}
& \sum_{n=1}^{\infty} \lambda_{n+1}\left(a_{1}^{\lambda_{1} v_{1}} a_{2}^{\lambda_{2} v_{2}} \cdots a_{n}^{\lambda_{n} v_{n}}\right)^{t / \Lambda_{n}} \\
& \quad \leq \frac{t e^{p / t}}{p} \sum_{n=1}^{\infty}\left(\frac{\Lambda_{n}^{\alpha}\left(\Lambda_{n}-\lambda_{n} v_{n} \beta\right)}{\Lambda_{n}\left(\Lambda_{n}+\lambda_{n} v_{n}\right)^{\alpha}}\right)^{p / t} \lambda_{n} v_{n} a_{n}^{p} \Lambda_{n}^{(p-t) / t}\left(\sum_{k=1}^{n} \lambda_{k} v_{k}\left(c_{k} a_{k}\right)^{p}\right)^{(t-p) / p}
\end{aligned}
$$

where

$$
c_{k}=\left[\frac{\left(\Lambda_{k}^{(1-t / p)} \Lambda_{k+1}\right)^{\Lambda_{k}} v_{k}^{\Lambda_{k-1}}}{\left(\Lambda_{k-1}^{(1-t / p)} \Lambda_{k}\right)^{\Lambda_{k-1}} v_{k+1}^{\Lambda_{k}}}\right]^{1 / \lambda_{k} v_{k} t}
$$

and $\alpha, \beta$ satisfy $0 \leq \alpha \leq 1 / \ln 2-1,0 \leq \beta \leq 1-2 / e$, and $e \beta+2^{1+\alpha}=e$.

Proof. The proof is almost the same as in Theorem 2.1. By the power mean inequality [11, page 15], we have (2.4) for $\alpha_{m} \geq 0, p>0$, and $q_{m}>0(m \in \mathbb{N})$ with $\sum_{m=1}^{n} q_{m}=1$. By (2.4), we obtain

$$
\left(\alpha_{1}^{q_{1}} \alpha_{2}^{q_{2}} \cdots \alpha_{n}^{q_{n}}\right)^{t} \leq\left(\sum_{m=1}^{n} q_{m} \alpha_{m}^{p}\right)^{t / p}
$$

for $t>0$. Taking $c_{m}>0, \alpha_{m}=c_{m} a_{m}$, and $q_{m}=\lambda_{m} v_{m} / \Lambda_{n}$, we have

$$
\left(\left(c_{1} a_{1}\right)^{\lambda_{1} v_{1} / \Lambda_{n}}\left(c_{2} a_{2}\right)^{\lambda_{2} v_{2} / \Lambda_{n}} \cdots\left(c_{n} a_{n}\right)^{\lambda_{n} v_{n} / \Lambda_{n}}\right)^{t} \leq\left(\sum_{m=1}^{n} \frac{\lambda_{m} v_{m}}{\Lambda_{n}}\left(c_{m} a_{m}\right)^{p}\right)^{t / p}
$$


Using the above inequality, we have

$$
\begin{aligned}
\sum_{n=1}^{\infty} \lambda_{n+1}\left(a_{1}^{\lambda_{1} v_{1}} a_{2}^{\lambda_{2} v_{2}} \cdots a_{n}^{\lambda_{n} v_{n}}\right)^{t / \Lambda_{n}} & \\
& \left.=\sum_{n=1}^{\infty} \lambda_{n+1} \frac{\left(\left(c_{1} a_{1}\right)^{\lambda_{1} v_{1} / \Lambda_{n}}\left(c_{2} a_{2}\right)^{\lambda_{2} v_{2} / \Lambda_{n}} \cdots\left(c_{n} a_{n}\right)^{\lambda_{n} v_{n} / \Lambda_{n}}\right)^{t}}{\left(c_{1}^{\lambda_{1} v_{1}} c_{2}^{\lambda_{2} v_{2}} \cdots c_{n}^{\lambda_{n} v_{n}}\right)^{t / \Lambda_{n}}}\right)\left(\sum_{m=1}^{n} \frac{\lambda_{m} v_{m}}{\Lambda_{n}}\left(c_{m} a_{m}\right)^{p}\right)^{t / p} . \\
& \leq \sum_{n=1}^{\infty}\left(\frac{\lambda_{n+1}}{\left(c_{1}^{\lambda_{1} v_{1}} c_{2}^{\lambda_{2} v_{2}} \cdots c_{n}^{\lambda_{n} v_{n}}\right)^{t / \Lambda_{n}}} .\right.
\end{aligned}
$$

By using the inequality (see $[4,12])$

$$
\left(\sum_{m=1}^{n} z_{m}\right)^{t} \leq t \sum_{n=1}^{\infty} z_{m}\left(\sum_{k=1}^{m} z_{k}\right)^{t-1}
$$

where $t \geq 1$ is constant and $z_{m}>0(n \in \mathbb{N})$, it is easy to observe that

$$
\frac{1}{\Lambda_{n}^{t / p}}\left(\sum_{m=1}^{n} \lambda_{m} \nu_{m}\left(c_{m} a_{m}\right)^{p}\right)^{t / p} \leq \frac{t}{p \Lambda_{n}^{t / p}} \sum_{m=1}^{n} \lambda_{m} \nu_{m}\left(c_{m} a_{m}\right)^{p}\left(\sum_{k=1}^{m} \lambda_{k} v_{k}\left(c_{k} a_{k}\right)^{p}\right)^{(t-p) / p}
$$

for $0<p \leq t$. Then, by (2.24) and (2.26), we obtain

$$
\begin{aligned}
& \sum_{n=1}^{\infty} \lambda_{n+1}\left(a_{1}^{\lambda_{1} v_{1}} a_{2}^{\lambda_{2} v_{2}} \cdots a_{n}^{\lambda_{n} v_{n}}\right)^{t / \Lambda_{n}} \\
& \leq \frac{t}{p} \sum_{n=1}^{\infty}\left(\frac{\lambda_{n+1}}{\Lambda_{n}^{t / p}\left(c_{1}^{\lambda_{1} v_{1}} c_{2}^{\lambda_{2} v_{2}} \cdots c_{n}^{\lambda_{n} v_{n}}\right)^{t / \Lambda_{n}}}\right) \sum_{m=1}^{n} \lambda_{m} v_{m}\left(c_{m} a_{m}\right)^{p}\left(\sum_{k=1}^{m} \lambda_{k} v_{k}\left(c_{k} a_{k}\right)^{p}\right)^{(t-p) / p} .
\end{aligned}
$$

Choosing $c_{1}^{\lambda_{1} v_{1}} c_{2}^{\lambda_{2} v_{2}} \cdots c_{n}^{\lambda_{n} v_{n}}=\left(\Lambda_{n}^{(1-t / p)} \Lambda_{n+1} v_{n+1}^{-1}\right)^{\Lambda_{n} / t}(n \in \mathbb{N})$ and setting $\Lambda_{0}=1$, from (2.19), it follows that

$$
\begin{aligned}
c_{n} & =\left(\frac{\left(\Lambda_{n}^{(1-t / p)} \Lambda_{n+1} v_{n+1}^{-1}\right)^{\Lambda_{n} / t}}{\left(\Lambda_{n-1}^{(1-t / p)} \Lambda_{n} v_{n}^{-1}\right)^{\Lambda_{n-1} / t}}\right)^{1 / \lambda_{n} v_{n}} \\
& =\left(\frac{\Lambda_{n+1} v_{n}^{\left(\Lambda_{n-1} / \Lambda_{n}\right)}}{\Lambda_{n-1}^{(1-t / p)\left(\Lambda_{n-1} / \Lambda_{n}\right)} \Lambda_{n}^{t / p} v_{n+1}}\right)^{\Lambda_{n} / \lambda_{n} v_{n} t} \Lambda_{n}^{1 / t} \\
& \leq\left(1+\frac{\lambda_{n} v_{n}}{\Lambda_{n}}\right)^{\Lambda_{n} / \lambda_{n} v_{n} t} \Lambda_{n}^{1 / t} .
\end{aligned}
$$


This implies that

$$
\begin{aligned}
& \sum_{n=1}^{\infty} \lambda_{n+1}\left(a_{1}^{\lambda_{1} v_{1}} a_{2}^{\lambda_{2} v_{2}} \cdots a_{n}^{\lambda_{n} v_{n}}\right)^{t / \Lambda_{n}} \\
& \leq \frac{t}{p} \sum_{m=1}^{n} \lambda_{m} v_{m}\left(c_{m} a_{m}\right)^{p} \sum_{n=m}^{\infty} \frac{\lambda_{n+1} v_{n+1}}{\Lambda_{n} \Lambda_{n+1}}\left(\sum_{k=1}^{m} \lambda_{k} v_{k}\left(c_{k} a_{k}\right)^{p}\right)^{(t-p) / p} \\
& \leq \frac{t}{p} \sum_{m=1}^{n}\left(1+\frac{\lambda_{m} v_{m}}{\Lambda_{m}}\right)^{p \Lambda_{m} / \lambda_{m} v_{m} t} \lambda_{m} v_{m} a_{m}^{p} \Lambda_{n}^{p / t} \\
& \quad \times \sum_{n=m}^{\infty}\left(\frac{1}{\Lambda_{n}}-\frac{1}{\Lambda_{n+1}}\right)\left(\sum_{k=1}^{m} \lambda_{k} v_{k}\left(c_{k} a_{k}\right)^{p}\right)^{(t-p) / p} \\
& \leq \frac{t}{p} \sum_{m=1}^{\infty}\left(1+\frac{\lambda_{m} v_{m}}{\Lambda_{m}}\right)^{p \Lambda_{m} / \lambda_{m} v_{m} t} \lambda_{m} v_{m} a_{m}^{p} \Lambda_{m}^{(p-t) / t}\left(\sum_{k=1}^{m} \lambda_{k} v_{k}\left(c_{k} a_{k}\right)^{p}\right)^{(t-p) / p} .
\end{aligned}
$$

Hence, using inequality (2.12) [19, Lemma 3.1] for $x>1$, and $\alpha, \beta$ satisfy $0 \leq \alpha \leq$ $1 / \ln 2-1,0 \leq \beta \leq 1-2 / e$, and $e \beta+2^{1+\alpha}=e$, we have (2.20). Thus Theorem 2.10 is proved.

REMARK 2.11. Theorem 2.10 reduces to Theorem 2.1 when $t=1$. Hence, inequality (2.20) is a new generalization of Hardy's inequality. Taking $v_{n}=1(n \in \mathbb{N})$ in Theorem 2.10, we obtain a result corresponding to [10, Theorem 2.6] with a weak condition for $\lambda_{n}(n \in \mathbb{N})$. Also assuming that $\lambda_{n}=1$ in Theorem 2.10, we have an extension of [10, Corollary 2.7] as in the following corollary.

COROLLARY 2.12. Let $v_{n}>0, a_{n} \geq 0, \Lambda_{n}=\sum_{m=1}^{n} v_{m}(n \in \mathbb{N})$, and $0<\sum_{n=1}^{\infty} v_{n} a_{n}<\infty$ for $0<p \leq t<\infty$. If

$$
\frac{\Lambda_{n+1} \Lambda_{n}}{\Lambda_{n}+\nu_{n}} \leq \Lambda_{n-1}^{((p-t) / p)\left(\Lambda_{n} / \Lambda_{n}\right)} \Lambda_{n}^{t / p} v_{n}^{-\left(\Lambda_{n-1} / \Lambda_{n}\right)} v_{n+1},
$$

then

$$
\begin{aligned}
& \sum_{n=1}^{\infty}\left(a_{1}^{\nu_{1}} a_{2}^{\nu_{2}} \cdots a_{n}^{\nu_{n}}\right)^{t / \Lambda_{n}} \\
& \quad \leq \frac{t e^{p / t}}{p} \sum_{n=1}^{\infty}\left(\frac{\Lambda_{n}^{\alpha}\left(\Lambda_{n}-\nu_{n} \beta\right)}{\Lambda_{n}\left(\Lambda_{n}+\nu_{n}\right)^{\alpha}}\right)^{p / t} v_{n} a_{n}^{p} \Lambda_{n}^{(p-t) / t}\left(\sum_{k=1}^{n} v_{k}\left(c_{k} a_{k}\right)^{p}\right)^{(t-p) / p},
\end{aligned}
$$

where

$$
c_{k}=\left(\frac{\left(\Lambda_{k}^{(1-t / p)} \Lambda_{k+1}\right)^{\Lambda_{k}} v_{k}^{\Lambda_{k-1}}}{\left(\Lambda_{k}^{(1-t / p)} \Lambda_{k}\right)^{\Lambda_{k-1}} v_{k+1}^{\Lambda_{k}}}\right)^{1 / v_{k} t}
$$


COROLLARY 2.13. Under the assumptions of Theorem 2.10, if $\alpha=0, \beta=1-2 / e$, then

$$
\begin{aligned}
& \sum_{n=1}^{\infty} \lambda_{n+1}\left(a_{1}^{\lambda_{1} v_{1}} a_{2}^{\lambda_{2} v_{2}} \cdots a_{n}^{\lambda_{n} v_{n}}\right)^{t / \Lambda_{n}} \\
& \quad \leq \frac{t e^{p / t}}{p} \sum_{n=1}^{\infty}\left(1-\frac{\lambda_{n} \nu_{n}(1-2 / e)}{\Lambda_{n}}\right)^{p / t} \lambda_{n} v_{n} a_{n}^{p} \Lambda_{n}^{(p-t) / t}\left(\sum_{k=1}^{n} \lambda_{k} v_{k}\left(c_{k} a_{k}\right)^{p}\right)^{(t-p) / p},
\end{aligned}
$$

where

$$
c_{k}^{\lambda_{k} v_{k} t}=\frac{\left(\Lambda_{k}^{(1-t / p)} \Lambda_{k+1}\right)^{\Lambda_{k}} v_{k}^{\Lambda_{k-1}}}{\left(\Lambda_{k-1}^{(1-t / p)} \Lambda_{k}\right)^{\Lambda_{k-1}} v_{k+1}^{\Lambda_{k}}} .
$$

COROLLARY 2.14. Under the assumptions of Theorem 2.1, if $\alpha=1 / \ln 2-1, \beta=0$, then

$$
\begin{aligned}
& \sum_{n=1}^{\infty} \lambda_{n+1}\left(a_{1}^{\lambda_{1} v_{1}} a_{2}^{\lambda_{2} v_{2}} \cdots a_{n}^{\lambda_{n} v_{n}}\right)^{t / \Lambda_{n}} \\
& \quad \leq \frac{t e^{p / t}}{p} \sum_{n=1}^{\infty}\left(\left(1+\frac{\lambda_{n} v_{n}}{\Lambda_{n}}\right)^{1-1 / \ln 2}\right)^{p / t} \lambda_{n} v_{n} a_{n}^{p} \Lambda_{n}^{(p-t) / t}\left(\sum_{k=1}^{n} \lambda_{k} v_{k}\left(c_{k} a_{k}\right)^{p}\right)^{(t-p) / p},
\end{aligned}
$$

where (2.34) holds.

REMARK 2.15. Corollaries 2.13 and 2.14 are new refinements and generalizations of [10, Corollaries 2.8 and 2.9], respectively. Further, taking $v_{n}=1(n \in \mathbb{N})$ in Corollaries 2.13 and 2.14, we obtain results corresponding to [10, Corollaries 2.8 and 2.9] with a weaker condition for $\lambda_{n}(n \in \mathbb{N})$.

\section{REFERENCES}

[1] H. Alzer, On Carleman's inequality, Portugal. Math. 50 (1993), no. 3, 331-334.

[2] _ _ Refinement of a Carleman-type inequality, Studia Sci. Math. Hungar. 32 (1996), no. 3-4, 361-366.

[3] _ A refinement of Carleman's inequality, J. Approx. Theory 95 (1998), no. 3, 497499.

[4] G. S. Davies and G. M. Petersen, On an inequality of Hardy's. II, Quart. J. Math. Oxford Ser. (2) 15 (1964), 35-40.

[5] G. Hardy, Note on a theorem of Hilbert, Math. Zeitschr. 6 (1920), 314-317.

[6] G. H. Hardy, J. E. Littlewood, and G. Pólya, Inequalities, Cambridge University Press, London, 1952.

[7] D. Y. Hwang and G. S. Yang, Note on discrete Hardy's inequality, Tamkang J. Math. 21 (1990), no. 4, 333-336.

[8] Y.-H. Kim, Refinements and extensions of an inequality, J. Math. Anal. Appl. 245 (2000), no. 2, 628-632.

[9] _ On Carleman's inequality and its improvement, Korean J. Comput. Appl. Math. 8 (2001), 1021-1026. 
[10] _ Refinements of Carleman's inequality and its generalizations, Indian J. Pure Appl. Math. 33 (2002), no. 12, 1775-1784.

[11] D. S. Mitrinović, J. E. Pečarić, and A. M. Fink, Classical and New Inequalities in Analysis, Mathematics and Its Applications, East European Series, vol. 61, Kluwer Academic Publishers, Dordrecht, 1993.

[12] J. Németh, Generalizations of the Hardy-Littlewood inequality, Acta Sci. Math. (Szeged) 32 (1971), 295-299.

[13] B. G. Pachpatte, A note on Copson's inequality involving series of positive terms, Tamkang J. Math. 21 (1990), no. 1, 13-19.

[14] P. Yan and G. Sun, A strengthened Carleman's inequality, J. Math. Anal. Appl. 240 (1999), no. 1, 290-293.

[15] B. Yang, On Hardy's inequality, J. Math. Anal. Appl. 234 (1999), no. 2, 717-722.

[16] B. Yang and L. Debnath, Some inequalities involving the constant e, and an application to Carleman's inequality, J. Math. Anal. Appl. 223 (1998), no. 1, 347-353.

[17] X. Yang, Approximations for constant e and their applications, J. Math. Anal. Appl. 262 (2001), no. 2, 651-659.

[18] _ On Carleman's inequality, J. Math. Anal. Appl. 253 (2001), no. 2, 691-694.

[19] B.-Q. Yuan, Refinements of Carleman's inequality, J. Inequal. Pure Appl. Math. 2 (2001), no. 2, Article 21, http://jipam.vu.edu.au/article.php?sid=137.

Dah-Yan Hwang: Center for General Education, Kuang Wu Institute of Technology, Peito, Taipei 11271, Taiwan

E-mail address: dyhuang@kwit.edu.tw 


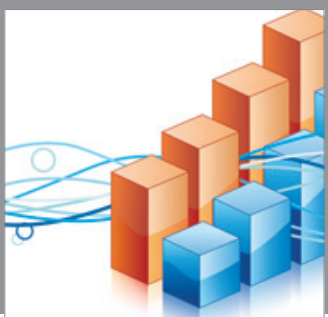

Advances in

Operations Research

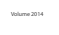

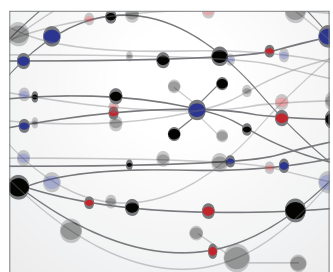

\section{The Scientific} World Journal
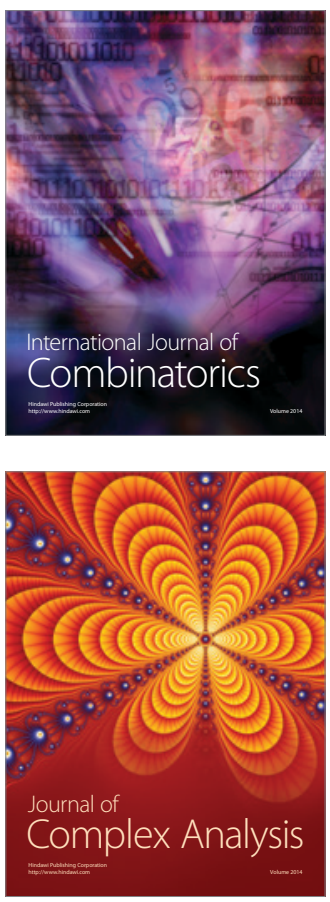

International Journal of

Mathematics and

Mathematical

Sciences
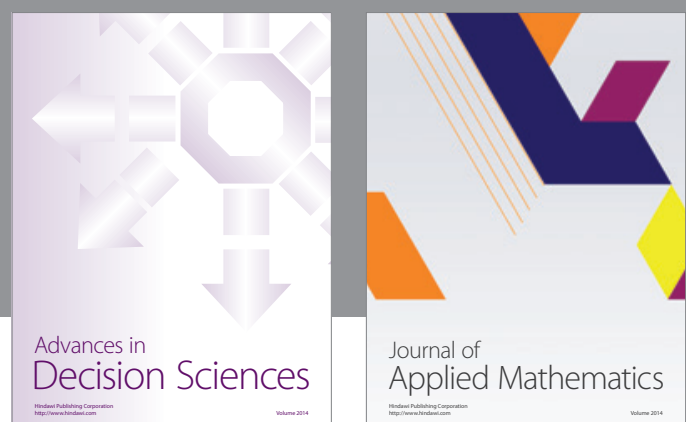

Journal of

Applied Mathematics
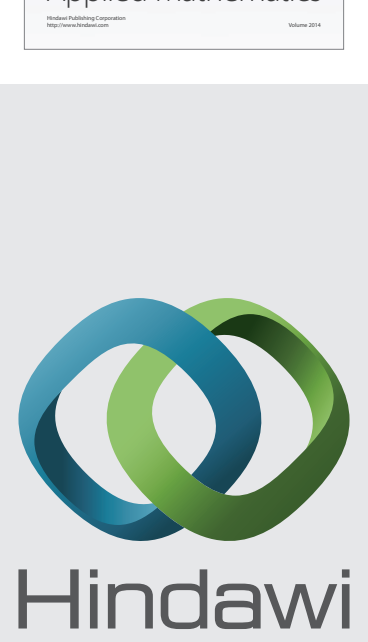

Submit your manuscripts at http://www.hindawi.com
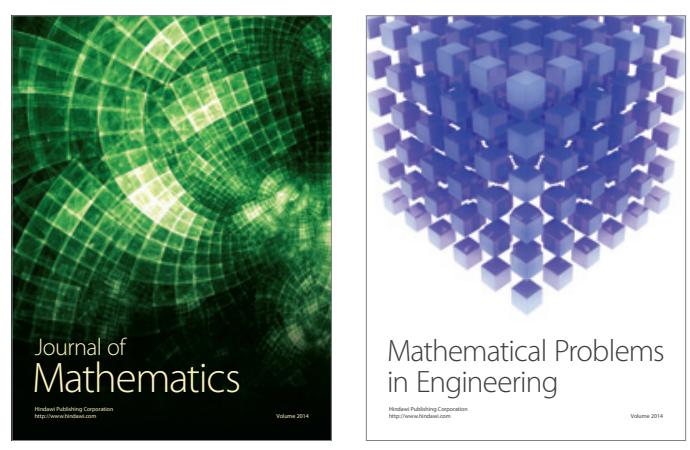

Mathematical Problems in Engineering
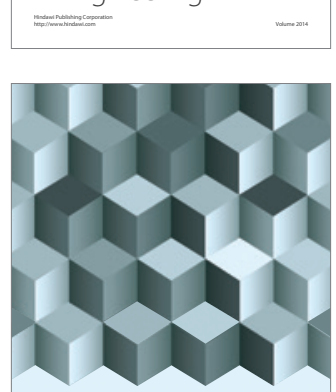

Journal of

Function Spaces
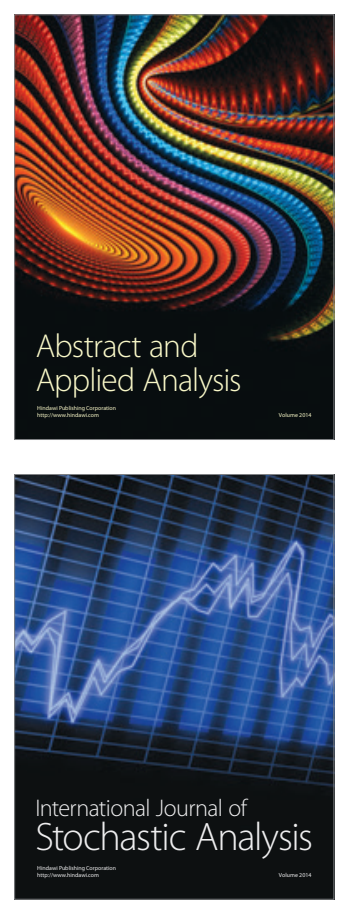

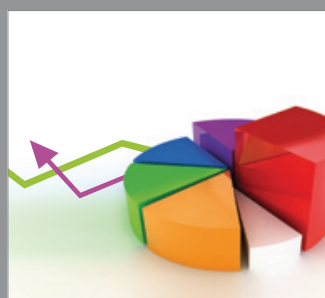

ournal of

Probability and Statistics

Promensencen
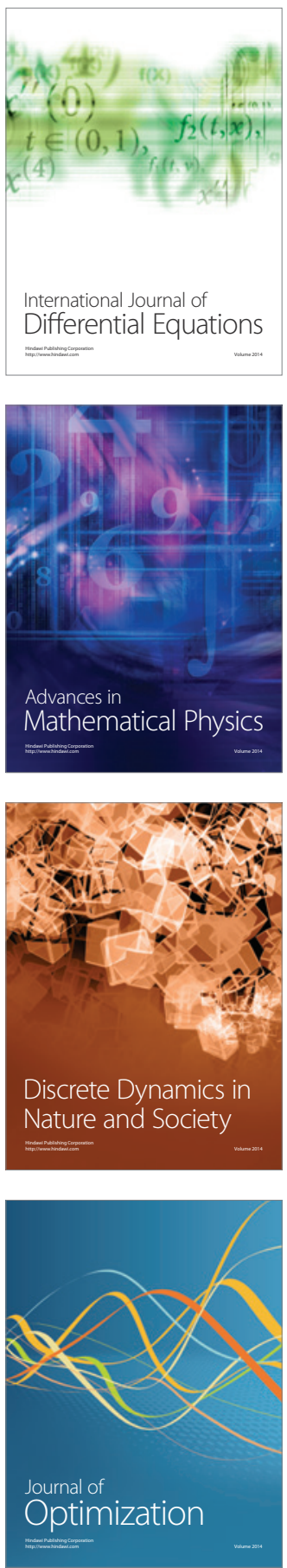